\title{
Modelling of Orthometric heights from Multi- Networks of GNSS/Precise Levelling in FCT, Abuja
}

\author{
Oluyori, P. D. , Ono, M. N. and Eteje, S. O. \\ Department of Surveying and Geoinformatics, Nnamdi Azikiwe University, Awka, Nigeria. \\ Corresponding Author: dareoluyori@gmail.com
}

\begin{abstract}
The geoid is used as a transformation linkage between ellipsoidal heights $(h)$ determined from DGPS observations and orthometric heights $(H)$. Widespread acceptability and adoption of GPS in local geospatial data acquisitions require the development of a local geoid model $(N)$ for use to obtain orthometric heights in the absence of a national geoid model. Geoid model can be developed by gravimetric approach; global geopotential model (GGM); geometric technique among others. The conventional approach to GPS measurements is the use of one base reference station for field measurements. It has several drawbacks e.g. in signal range/coverage, accuracy degradation of results, etc. Based on Grashof's law of stability of triangles, this study was therefore based on dual reference base stations to improve on DGPS signal range and stability of results. Pro-online matrix solver was applied to the least squares observation equations of the two modelled FCT surfaces (multi quadratic and bicubic) to determine polynomial coefficients. The geoid undulation was computed and orthometric height generated for production of a topographical plan at $1 \mathrm{~m}$ contour interval for elevation data in surveying, engineering and environmental applications. Skill $=1$ and bias $=0$ were computed to confirm the predictive capability of the models and that no bias/errors were introduced into the respective modelling exercise. Diagnostic test also confirmed the viability and feasibility of providing vertical datum surface for FCT by this approach. Standard deviation $(\sigma)$ as accuracy indicator was computed and the multi-quadratic model with $\sigma=11 \mathrm{~cm}$ was the better geoid surface for modelling of orthometric height in the FCT by the geometric method.
\end{abstract}

Keywords-Geoid undulation, Multiquadratic, Bicubic, Grashof's law, Orthometric height.

\section{INTRODUCTION}

The use of GNSS in orthometric height $(\mathrm{H})$ determination requires a geoid model $(\mathrm{N})$ to transform the observed ellipsoidal height (h). For global applications, global geoid models (EGM2008) have been developed to provide the geoid undulation. For small to medium-sized areas, global geoid model, according to Odera and Fukuda (2015) is too generalized and will lead to error in orthometric height if applied. Merry (2009) gave a value of $3 \mathrm{~m}$ in Central Mozambique when compared with EGM2008 values due to the use of generated gravity anomalies. Hence, this requires the development of local geoid models for the needs of GNSS user community in geospatial data acquisitions and applications.

Al-kragy et al. (2015) observed that a geoid model is a three dimensional (3D) geospatial model that defines the relationship between the ellipsoid and the geoid surfaces at a specific area. Eteje et al. (2018) defined geoid as the surface which coincides with that surface to which the oceans will conform over the entire earth if free www.ijeab.com to adjust to the combined effects of earth's mass attraction and the centrifugal force of earth's rotation. Methods of geoid undulation determination are namely:

(a). Gravity measurements for gravimetric geoid by solving general Stoke's integral formula by spherical harmonic expansion as given by Heiskanen and Moritz (1967):

$$
\begin{aligned}
& N=K \frac{K \delta m}{R G}-\frac{\delta W}{G}+\frac{R}{4 \pi G} \iint \Delta g S(\varphi) d \sigma \\
& S(\varphi)=1+\frac{1}{\sin \frac{\varphi}{2}}-6 \sin \frac{\varphi}{2}-5 \cos \varphi-
\end{aligned}
$$

$3 \cos \varphi \ln \left(\sin \frac{\varphi}{2}+\sin ^{2} \frac{\varphi}{2}\right)$

where the various parameters are as given in the literature.

Assuming that the mass of real earth is equal to the mass of the normal earth and the potential generated by 
two masses to be equal, the first two terms in equation (1) become zero i.e.

$$
N=\frac{R}{4 \pi G} \iint \Delta g S(\varphi) d \sigma
$$

The difficulty with Stoke's formula is that solution requires gravity data all over the earth which is impossible to achieve arising from the double integral in the formula. To overcome this, global geopotential models (GGM) were developed. These global models are inadequate for local applications and Odera et al. (2015) stated that they are too generalized to be useful for local applications and hence, for areas of limited sizes, a local geometric geoid model could be developed for orthometric data acquisitions.

\section{(b) Geometric Geoid Model}

This is developed for areas ranging from small to medium and computed directly from GPS based ellipsoidal height (h) and collocated with points of known orthometric heights $(\mathrm{H})$. From the relationship given by Kotsakis and Sideris (1999), Jekeli (2006), a linear relationship between $\mathrm{h}, \mathrm{H}$ and $\mathrm{N}$ where $\xi$ is deviation of the vertical and curvature of plumb line is $h=N+H+\xi$

Seker and Yildirin (2002) observed that at $\xi=1^{\prime \prime}$, the error incurred is $0.08 \mathrm{~mm}$ which is negligible, insignificant and of no practical consequence. Also, Nordin (2009) computed the effect of $\xi=11^{\prime \prime}$ as less than $1 \mathrm{~mm}$. Figure 1 shows the linear relationship between the heights. The combined interpretation and implication of the above values is that we can write with confidence that:

$$
N=h-H
$$

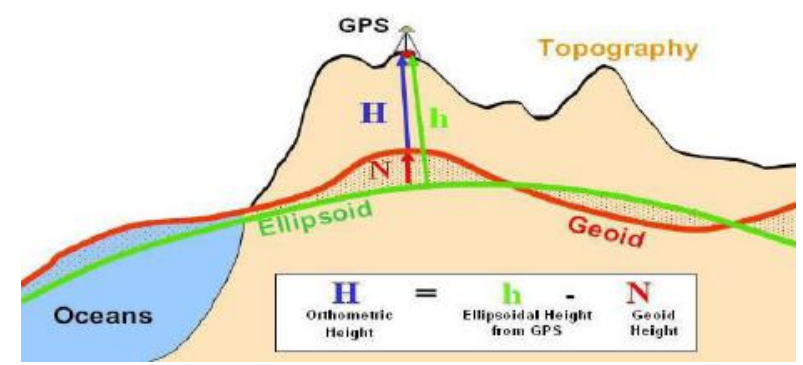

Fig. 1: Relationship between Orthometric, Geoid and Ellipsoidal Heights

Source: Ono (2013) and Eteje et al. (2018)

\subsection{Datum Ambiguity/Bias}

The vertical datum may be inconsistent due to measurement error and is evident in all three height components of $h, H$ and $N$. Let $\delta h, \delta N$ and $\delta H$ be the biases in each of the components, we can, therefore, write from equation (6)
$h=N+H$

that

$$
\begin{aligned}
& h+\delta h=(H+\delta H)+(N+\delta N) \\
& h=H+N+(\delta H+\delta N-\delta h) \\
& h=H+N+S
\end{aligned}
$$

where,

$$
S=(\delta H+\delta N-\delta h)
$$

By comparing $h=N+H+\xi$ with $h=H+N+S$, it can be shown that $S=\xi$. Hence $\xi=(\delta H+\delta N-\delta h)$ is insignificant. From the various values of $\xi$ computed by the above authors, the datum bias can therefore be taken as insignificant and hence negligible for low order survey and engineering applications and adequate for geometric geoid modelling (from $N=h-H$ ) and hence orthometric height determination from $H=h-N$. Milbert and Smith (1996) observed that the very small values of $S$ compared to $N$ support the direct conversion between ellipsoid and orthometric vertical datum even if they are not defined on a common reference. Geometric geoid model hence is adopted for modelling orthometric height in the provision of vertical datum for elevation data acquisition.

Kamaludin et al. (2005) observed that differential heighting method can be used to eliminate datum inconsistencies for topographical and engineering/environmental studies and applications. From $N=h-H$, interpolation of geoidal heights $(N)$ becomes feasible over interested points with an available GPS ellipsoidal and existing orthometric heights.

\subsection{Justification of Adopted Field Procedure}

Generally, in DGPS campaigns only one base reference station is adopted for observations in the relative approach. This method has limitations in coverage and accuracy is spatially degraded after certain distances beyond, for example, $10 \mathrm{~km}$ or over large areas. Martensson (2002) recommended the use of network that resembles a triangulation network in GPS campaigns where the aim is to obtain surface cover for geometric geoid modelling to ensure that no deterioration of results are experienced and hence it can be stated that the results from this study are highly stable and consistent since the FCT triangulation network was used for all measurements. Chang and Lin (1999) reported from studies using one and multiple base reference stations, that results obtained from the latter are more reliable and consistent achieving over $60 \%$ improvement in values both in horizontal and vertical components using DGPS. 


\subsection{Stability of Shapes}

A triangle is the simplest of closed figures in two dimensions and described as the strongest geometrical shape and most stable too because of its inherent structural characteristics. For example, a square is capable of becoming a parallelogram whereas a triangle is only capable of being a triangle. The explanation given to why the triangle is more stable than other shapes is that it only takes three points to define a plane. By adding any point to the plane will make it harder and harder for it to be stable. Also, no matter where the vertices of the triangle lie, they will always define a plane and hence triangles are both stable and rigid. Grashof's relationship can be used to compute geometrical stability of figure from Quora http:// www.quora.com as:

$n=3(L-1)-2 J-h$

where,

$n=$ degree of freedom

$L$ is number of links; $J$ is number of joints ; $h$ is number of higher points. If $n=0$, there is geometrical stability of results for a triangular geometry formed with two base reference receivers and one rover station as shown in Figure 2

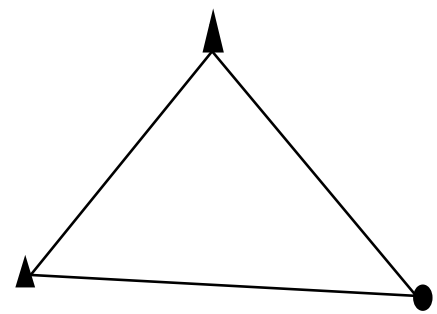

Fig. 2: Base Reference/Receiver (P)
From Figure 2, for a triangle, $L=3, J=3, h=0 ; n=3$ (3$1)-2 \times 3-0=0$. This $(n=0)$ implies the adopting of triangular geometry for GPS observations, geometrical stability of results is achieved. For a line used as the conventional method of GPS relative technique of one base reference station and one rover position as shown in Figure 3, i.e. computed to be $\mathrm{n}=-4$ to show that line used for field observation may not produce stable results.

\section{$\Delta \longrightarrow \Delta$ \\ Base/Ref. \\ $\mathrm{L}=1$ \\ Rover}

Fig. 3: A line

For a pentagonal geometry, $L=5, J=5, h=0$, therefore $n$ $=2$ to indicate and imply that a deformed and in stable shape that may affect the data.

\section{METHOOLOGY}

\subsection{GPS Field Observations}

Dual base reference stations were used to determine the ellipsoidal heights of the observed controls used as rover positions with DGPS receivers and accessories. Three online post processing software was used to process for the ellipsoidal height and the arithmetic means of the ellipsoidal heights were computed. The average ellipsoidal heights of each point was used with the existing orthometric height to determine the geoid undulation of each control point, equation (5). See the results in Table 1

Table 1: Average Ellipsoidal Heights, Existing Orthometric Heights and Computed Geoid Undulations

\begin{tabular}{|c|c|c|c|c|c|}
\hline & \multicolumn{3}{|c|}{ COORDINATE REGISTER VALUE } & \multirow{2}{*}{$\begin{array}{c}\text { POST } \\
\text { PROCESSED } \\
\text { AVERAGE h } \\
\text { (m) }\end{array}$} & \multirow[b]{2}{*}{$\begin{array}{c}\text { UNDULATION, } \\
\text { N=h-H (m) }\end{array}$} \\
\hline $\begin{array}{l}\text { CONTROL } \\
\text { POINTS }\end{array}$ & $\begin{array}{l}\text { EASTINGS } \\
\quad(\mathrm{m})(\mathrm{e}) \mathrm{X}\end{array}$ & $\begin{array}{l}\text { NORTHINGS (m) } \\
\text { (n)Y Y }\end{array}$ & $\begin{array}{c}\text { EXISTING } \\
\text { ORTHO. } \\
\text { HEIGHTS } \\
\text { H (m) }\end{array}$ & & \\
\hline FCC11S & 331888.114 & 998442.043 & 485.447 & 509.396 & 23.949 \\
\hline FCT260P & 255881.175 & 993666.807 & 201.944 & 224.74 & 22.787 \\
\hline FCT103P & 340639.766 & 998375.578 & 532.558 & 556.836 & 24.278 \\
\hline FCT12P & 333743.992 & 1008308.730 & 735.707 & 760.192 & 24.485 \\
\hline FCT19P & 337452.408 & 996344.691 & 635.644 & 659.824 & 24.18 \\
\hline FCT2168S & 310554.927 & 1009739.930 & 431.087 & 455.274 & 24.187 \\
\hline FCT24P & 322719.776 & 1001884.850 & 453.804 & 477.987 & 24.183 \\
\hline FCT276P & 351983.716 & 1025998.314 & 625.572 & 649.848 & 24.276 \\
\hline FCT4154S & 329953.882 & 1003831.280 & 476.981 & 501.232 & 24.251 \\
\hline FCT4159S & 326124.422 & 1003742.860 & 452.230 & 476.553 & 24.323 \\
\hline FCT66P & 299148.035 & 998114.283 & 297.111 & 321.115 & 24.004 \\
\hline FCT9P & 329821.512 & 1007612.091 & 497.253 & 521.693 & 24.440 \\
\hline FCT35P & 322183.380 & 992926.363 & 427.171 & 451.299 & 24.128 \\
\hline FCT57P & 303234.270 & 992916.402 & 323.844 & 347.795 & 23.951 \\
\hline FCT4028S & 330164.634 & 1001388.240 & 449.592 & 473.942 & 24.35 \\
\hline FCT53P & 308943.361 & 993406.773 & 351.943 & 375.955 & 24.012 \\
\hline FCT4652S & 329441.767 & 997474.808 & 462.711 & 487.113 & 24.402 \\
\hline
\end{tabular}




\begin{tabular}{|c|c|c|c|c|c|}
\hline FCT162P & 270791.291 & 934625.533 & 189.696 & 215.091 & 25.395 \\
\hline FCT130P & 330982.584 & 952889.869 & 695.608 & 719.383 & 23.775 \\
\hline FCT2327S & 282526.612 & 973821.470 & 183.287 & 207.482 & 24.195 \\
\hline FCT2652S & 271370.273 & 945385.429 & 138.952 & 163.741 & 24.789 \\
\hline FCT2656S & 272644.591 & 941062.460 & 204.724 & 229.229 & 24.505 \\
\hline FCT83P & 332954.205 & 987231.606 & 568.752 & 592.819 & 24.067 \\
\hline XP382 & 284074.729 & 983364.863 & 274.586 & 298.390 & 23.804 \\
\hline
\end{tabular}

\subsection{Polynomial Surfaces}

The two polynomial surfaces considered to represent/model the FCT continuous vertical reference surface are: i) multiquadratic and ii) bicubic.

1. Multi - quadratic model (nine parameters) from Sanlioglu et al. (2009)

$$
\begin{aligned}
N= & a_{0}+a_{1} X+a_{2} Y+a_{3} X^{2}+a_{4} Y^{2}+ \\
& a_{5} X Y+a_{6} X^{2} Y+a_{7} X Y^{2}+a_{8} X^{2} Y^{2}
\end{aligned}
$$

Multi-quadratic interpolation according to Yanalak and Baykal (2001) is an analytical method of representing irregular surfaces that involve the summation of quadratic surfaces. Kirici and Sisman (2017) stated that even if the reference points are not homogeneously distributed, the results of surface modelling are barely affected. This is particularly applicable to the present studies with reference to the lopsided distribution of controls selected (after reconnaissance surveys) for use in geometric geoid development.

2. Bi- cubic model (third-order polynomial)

$$
\begin{aligned}
N= & a_{00}+a_{10} X+a_{01} Y+a_{20} X^{2}+ \\
& a_{11} X Y+a_{02} Y^{2}+a_{30} X^{3}+ \\
& a_{21} X^{2} Y+a_{12} X Y^{2}+a_{03} Y^{3}
\end{aligned}
$$

Where,

$$
Y=A B S\left(y-y_{o}\right)
$$

$X=A B S\left(x-x_{o}\right)$

$y=$ Northing coordinate of observed station

$x=$ Easting coordinate of observed station

$y_{o}=$ Northing coordinate of the origin (average of the northing coordinates)

$x_{o}=$ Easting coordinate of the origin (average of the easting coordinates)

\subsection{Least Squares Equation and Solutions}

Observation equation was formed for each point and solved to determine the polynomial coefficients $\mathrm{X}$ from the observation equation generally as given by Ono et al. (2004):

$V=A X-L$

where,
$A=$ coefficient matrix

$X=$ vector of unknown parameters/coefficients

$L=$ geoid undulations

Applying least squares principles, the solution is given by

$$
X=\left(A^{T} A\right)^{-1}\left(A^{T} L\right) \text { for unit weight }
$$

Unit weight $(\mathrm{W}=1)$ is assumed due to equal reliability of observations.

The geoidal undulation of at least six points must be known within the study area to enable redundancies for the robustness of least squares solution. In this study, twenty-four (24) points with both ellipsoidal and orthometric heights are known. The model parameters determined from least squares solutions are:

Multi - Quadratic Model Parameters

$$
X=\left(\begin{array}{l}
a_{o} \\
a_{1} \\
a_{2} \\
a_{3} \\
a_{4} \\
a_{5} \\
a_{6} \\
a_{7} \\
a_{8}
\end{array}\right)=\left(\begin{array}{c}
24.2248901210000000000 \\
-0.00002409340580587179 \\
-0.00008013620770038382 \\
0.00000000009699046795 \\
0.00000000370280953876 \\
0.00000001166702184889 \\
-0.00000000000021600943 \\
-0.00000000000045716237 \\
0.00000000000000000886
\end{array}\right)
$$

Bicubic Model Parameters

$$
X=\left(\begin{array}{l}
a_{o} \\
a_{1} \\
a_{2} \\
a_{3} \\
a_{4} \\
a_{5} \\
a_{6} \\
a_{7} \\
a_{8} \\
a_{9}
\end{array}\right)=\left(\begin{array}{c}
23.50081592604167925515 \\
0.00004395285221636346 \\
0.00009105227330487502 \\
-0.00000000156204910634 \\
-0.00000000352164634358 \\
-0.00000000178532065159 \\
0.00000000000004116279 \\
0.00000000000002433215 \\
0.00000000000000729517 \\
0.00000000000002928003
\end{array}\right)
$$

Standard deviation of observations $(\sigma)$ was computed using (15):

$\sigma=\sqrt{\frac{\sum v^{2}}{(n-1)}}$

where, 
$v=$ residual $=$ difference between model and known geoid height.

$n=$ number of points.

2.4 Interpolation of Geoid and Orthometric Height Modelling

Microsoft Excel program was developed to interpolate the geoid undulation and hence model the orthometric height for each point within the study area. The $x, y$ and h are input into the Microsoft Excel program developed to interpolate both geoid and orthometric heights. The modelled orthometric heights were then compared with their corresponding existing orthometric heights of the controls and the standard deviation was computed from equation (15) as $\sigma_{\text {multi }}=11 \mathrm{~cm}$ and $\sigma_{\text {Bicubic }}=14 \mathrm{~cm}$.

\subsection{Hypothesis Testing for Comparison of} Orthometric Height

The null hypothesis is given by $H_{0}$ while the alternative is $H_{1}$ and is formulated as follows:

$H_{o}: \mathrm{R}=0$, no relationship between $H_{\text {Multiquadatic }}$ and

$$
H_{\text {MSL }}
$$

$H_{1}: \mathrm{R} \neq 0$, there is a relationship between

$$
H_{\text {Multiquadatic }} \text { and } H_{\mathrm{MSL}}
$$

Significance level $\alpha=0.05$ i.e. $95 \%$ confidence level.

Decision rule: reject $H_{o}$ if $\mid$ computed $t \mid>t_{20,0.05}$ from $t$ - distribution table.

Scenario: $H_{\text {Multiquadatic }}$ and $H_{\text {MSL }}$

The statistical significance of the relationship was computed by $t$-test statistics formula given in janda.org/c/10/lectures/topic/L as:

$t=R \sqrt{(n-2) /\left(1-R^{2}\right)}$

In the case of $H_{\text {Multiquadatic }}$ and $H_{\mathrm{MSL}}$, the computed $t=$ 0 while table $t=1.717$. From the decision rule above, we reject $H_{o}$ i.e. the existing heights do not have any correlation with the modelled heights. Hence $H_{1}$ is accepted and further, it may be an indication of coincidence of the two surfaces but referenced to different reference datum, the geoid and the mean sea level. Height values based on the geoid (multi-quadratic or bi-cubic models) are the desired orthometric heights and is the primary goal of this study in FCT for height modernization according to Nwilo (2013).

\subsection{Evaluation of Surface Fitting Techniques}

Alevzakou and Lambrou (2011) stressed the need to determine if a surface of higher degree is necessary in geometric geoid modelling by using the relationship given as:

$$
\left(r_{1} \sigma_{2} / r_{2} \sigma_{1}\right) \leq F_{1} r_{2}
$$

Where,

$r_{1}, r_{2}=$ degrees of freedom of the smaller degree surface and the greater surface respectively.

$\sigma_{1}, \sigma_{2}=$ standard deviation of the two surfaces respectively.

$F_{1}, r_{2}=\mathrm{F}$ distribution for one degree difference between the tested surfaces $\sigma_{1}=0.109959231 \mathrm{~m}$ $\sigma_{2}=0.135719119 \mathrm{~m} \quad r_{1}=15$ (multi-quadratic surface), $r_{2}=14$ (bi-cubic surface).

The decision rule is if $\left(r_{1} \sigma_{2} / r_{2} \sigma_{1}\right) \leq F_{1} r_{2}$, then no higher surface is needed for geometric geoid modelling of FCT. The Computed value of $\left(r_{1} \sigma_{2} / r_{2} \sigma_{1}\right)=$ 1.32242959925

$F_{1}, r_{2}$ from $\mathrm{F}$ Tables $=4.531$ (http://www.stat.ucla.edu/dinov).

From the relationship $\left(r_{1} \sigma_{2} / r_{2} \sigma_{1}\right)$ and $F$ distribution $F_{1}, r_{2}$, since $1.32242959925<4.531$, no higher degree surface is needed for geometric geoid model in the FCT. This is an indication that either multi-quadratic or bicubic model can be used to model orthometric height although the multi-quadratic model performed better and could be taken as the optimum.

\subsection{Skill and Bias Estimates}

The skill parameter can be used as a measure of the model predictive capacity in relation to the observations. This skill parameter ranges from negative values to one with the corresponding value of one implying a total agreement between observations and the model results. The bias values computed as zero simply imply that the data used, equipment used and personal error did not show any bias whatsoever in this study. Bias and skill were calculated by the relationship given by Sutherland et al. (2004)

This also suggested that the selection and combination of equipment, personnel, field techniques and processing methods adopted yielded high quality data to produce the FCT geoid surface information as much as possible. Orthometric heights from the surface are hence based on geoid and compatible with GNSS technique and the adopted dual base reference stations technique.

2.8 Diagnostic Test for Multiquadratic and Bicubic Models 
To carry out a diagnostic test for the predictive ability of the models in orthometric height modelling as stated in Sinha and Prasad (1979), the computation was carried out using $1.98 / \sqrt{N}$ where $N$ is the number of stations $=24$ in this study to compare with chi-squares table values. The decision rule is if $1.98 / \sqrt{N}<\chi^{2}$, then models are satisfactory at $95 \%$ confidence level. In this study, $1.98 / \sqrt{N}=0.404$. Using the Chi squares $\left(\chi^{2}\right)$ test at the $95 \%$ degrees of freedom (d.o.f), we have for multiquadratic model, degree of freedom $=15$, at $95 \%, \chi^{2}=24.996$; bicubic model, degree of freedom $=$ 14 at $95 \% \chi^{2}=23.685$. Since $0.404<24.996$ or 23.685 , the models proved satisfactory at $95 \%$ confidence limits for modelling orthometric heights from GNSS techniques as confirmed by the diagnostic tests.

\subsection{Application Areas and Importance of the Geoid} Model

Applications of geoid are:

1. For transforming GPS ellipsoidal heights (h) to orthometric height for practical surveying and engineering applications.

2. An important part of a National Geodetic Data Infrastructure (NGDI).
3. The geoidal map can also be used to interpolate for geoid heights at any point of interest in FCT.

4. This is useful where the conventional method of spirit levelling is costly, tedious, time-consuming and costly especially in highly urbanized areas.

The importance of the determined geoid models in orthometric height derivation are:-

1. Consistency and compatibility with GNSS technique is achieved with these models for orthometric height determination.

2. Orthometric heights can be interpolated for all points of interest within the FCT.

\section{DISCUSSION OF RESULTS}

\subsection{Plot of Geoid Undulation Against Controls}

Figure 5 presents the plots of multi-quadratic and bicubic geoid heights against control stations. This was done to show graphically the differences between the multiquadratic and the bi-cubic models' geoid undulations. It can be seen that the two surfaces are nearly coincident and identical from a visual inspection of Figure 5. This implies and confirms the interchangeability and acceptability of the two models for orthometric height determination within the FCT but attaching more weight to the multi-quadratic model. However, visual observations/interpretations are generally subjective.

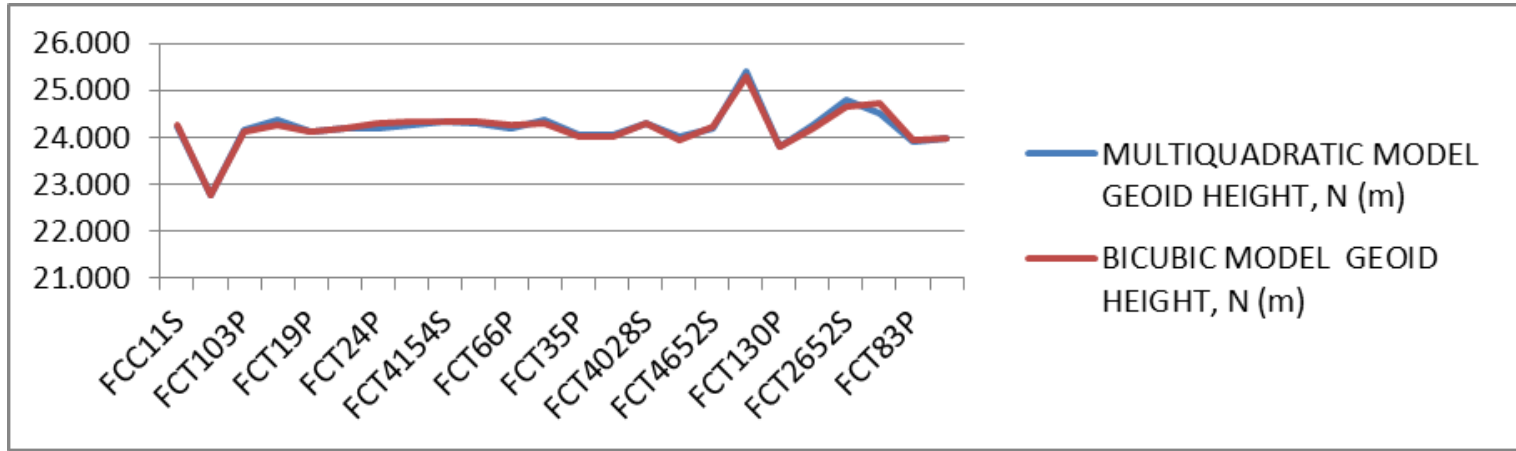

Fig. 5: Geoid Undulation Models from both Multi-Quadratic and Bi-Cubic Models.

\subsection{Similarity of Surfaces}

Figure 6 shows the plots of the multi-quadratic model, bicubic model and existing orthometric heights. This was also done to show graphically the similarity of the three surfaces. From Figure 6, it can be confirmed that the multi-quadratic model is more suitable and adequate as it is smoother for the modelling of orthometric heights in FCT by GNSS technique. 


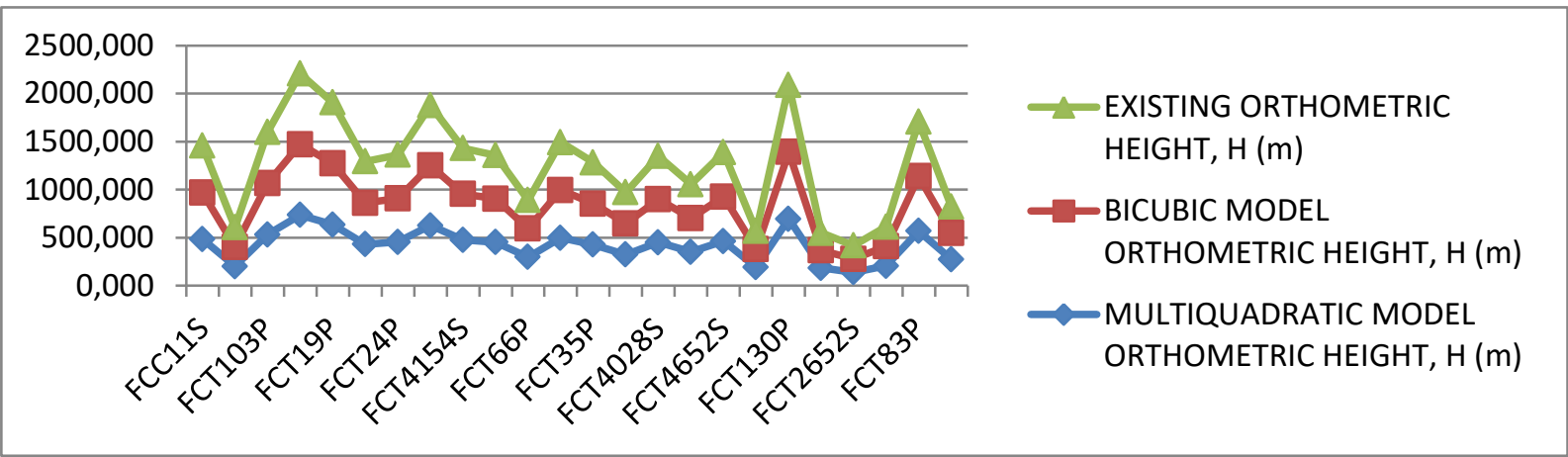

Fig. 6: Similarity Plots of the Three Surfaces

\section{CONCLUSIONS}

From the study, the following conclusions can be made:

1. Multiquadratic model takes good care of the lack of homogeneous distribution of selected controls in geoid modelling.

2. Coefficient of correlation $(R)$ and coefficient of determination $\left(R^{2}\right)$ values of $R=0.995 \mathrm{~m}$ and $\left(R^{2}\right)=99 \%$ respectively indicate the multiquadratic model has a high predictive ability at $95 \%$ confidence limits.

3. Dual base reference stations were adopted for data acquisition instead of conventional single base reference station. This enabled the stability of results by exploiting Grashof's law of stability of triangles.

4. The feasibility of developing a geoid model for GPS user community by GNSS/Levelling in FCT has been demonstrated as an alternative approach to conventional spirit levelling in orthometric height determination

\section{REFERENCES}

Alevizakolu, E and Lambrou, E. (2011). Fast and Convenient Determination of Geoid Undulation $(\mathrm{N})$ in Urban Area. FIG Working Week, Marrakech, Morroco,18-22 May 2011.

[1] Al-Kragy, E., Hosny, M and Dawod, G.M (2015). Investigating the Precision of Recent Global Geoid Models and Global Digital Elevation Models for Geoid Modelling in Egypt. Regional Conference on Surveying and Development, Sharm El-Sheik, Egypt, 3-6 October 2015.

[2] Chang, C. .C and Lin, S. H. (1999). Testing a Medium-Range DGPS Network for the Taiwan Area. The Journal of Navigation, Vol. 52, No. 2, pp $279-288$.

[3] Eteje, S. O., Ono, M. N. and Oduyebo, F. O. (2018). Practical Local Geoid Model Determination for Mean Sea Level Heights of Surveys and Stable Building Projects. IOSR Journal of Environmental Science, Toxicology and Food Technology (IOSR-
JESTFT), Vol. 12, No. 10.9790/2402-1206013037.

6, pp 30-37. DOI:

[4] Eteje, S.O., Oduyebo. O.F., and Olulade, S, A. (2018). Procedure for the Determination of Local Gravimetric-Geometric Geoid Model, International Journal of Advances in Scientific Research and Engineering, Vol. 4, No. 8 pp 206-214.

[5] Heiskanen, W.A and Moritz, H. (1967). Physical Geodesy, New York. W. H. Freeman \& Co., 364p.

[6] Jekeli, C. (2006). Geometric Reference Systems in Geodesy. Division of Geodesy and Geospatial Science, School of Earth Sciences, Ohio State University.

[7] Kamaludin, M. O., Sharum, S. and Azhair, M. (2005). Enhancement of Height System for Malaysia Using Space Technology. Faculty of Geoinformation Science and Engineering, University Teknologi, Malaysia.

[8] Kirici, U. and Sisiman, Y. (2017). The Comparison of the Adjustment Methods in Geoid Determination Method. FIG Working Week 2017.

[9] Kotsakis, C. and Sideris, M. G. (1999). On the Adjustment of Combined GPS/Levelling Geoid Networks. Journal of Geodesy, Vol. 73, No. 8, pp 412-421.

[10] Martensson. S. G. (2002). Height Determination by GPS - Accuracy with Respect to Different Geoid Models in Sweden, FIG XXII International Congress, Washington, 19 - 26 April 2002.

[11] Merry, C. (2009). EGM 2008 Evaluation for Africa. Newton's Bulletin, No.4, 2009.

[12] Milbert, D. G. and Smith, D. A. (1996). Converting GPS Height into NAVD88 Elevation with GEOID 96 Geoid Height Model. National Geodetic System, NOAA.

[13] Nordin, S. B. (2009). Height Modernization Using Fitted Geoid Models and MyRTKNET. Published M.Sc. Thesis, Faculty of Geoinformation Science and Engineering, Universiti Teknologi, Malaysia.

[14] Nwilo, P. C. (2013). Technological Advancement in Surveying and Mapping: The Nigerian Adaptation, FIG Working Week, Abuja Nigeria.

[15] Odera, P. A. and Fukuda, Y. (2015). Recovery of Orthometric Heights from Ellipsoidal Heights Using Offsets Method, Earth Planets and Space, Vol. 
67, No.1, pp 1-7. DOI 10.1186/s40623-015-0306$\mathrm{z}$.

[16] Ono, M. N. (2013). Advanced Geometric Geodesy. Unpublished Lecture Note of the Department of Surveying and Geoinformatics, Nnamdi Azikiwe University Awka.

[17] Ono, M. N., Igbokwe, J. I., Ojiakor, J. C. and Emengini, J. E. (2004). Three Dimensional Integration Approach for Adjusting National Geodetic Network for Satellite Mapping. Nigerian Institution of Surveyors, Garden City Benin.

[18] Sanlioglu, I., Maras, S. S. and Uysal, F. (2009). Determination of Orthometric Heights with RealTime Kinematic Surveying, Konya Sample, FIG Working Week.
[19] Seker, D. Z. and Yildrim, A. (2002). Orthometric Height Derivation from GPS Observations. FIG XXII International Congress, Washington D.C.USA, April 19-26 2002.

[20] Sinha, N. K. and Prisad, T. (1979). Some Stochastic Modelling Techniques and their Applications. Appl. Math. Modeling., Vol. 3.

[21] Sutherland, J., Pete, A. and Soulsby, R. (2004). Evaluating the Performance of Morphological Models. Coastal Engineering, Vol. 51, pp 917-939. DOI: 10.1016/j.coastaleng.2004.07.015

[22] Yanalak, M. and Baykal, O. (2001). Transformation of Ellipsoid Heights to Local Levelling Heights. Journal of Surveying Engineering, Vol. 127, pp. 1-14. 